\title{
A New Uncertainty Measure in Belief Entropy Framework
}

\author{
Moïse Digrais Mambé1,4, Tchimou N'Takpé ${ }^{2,4}$, Nogbou Georges Anoh ${ }^{3,4}$, Souleymane Oumtanaga ${ }^{1,4}$ \\ ${ }^{1}$ Institut National Polytechnique Félix Houphouët-Boigny, Yamoussoukro, Côte d'Ivoire \\ ${ }^{2}$ Université Nangui Abrogoua, Abidjan, Côte d'Ivoire \\ ${ }^{3}$ Université Virtuelle de Côte d'Ivoire, Abidjan \\ ${ }^{4}$ Laboratoire de Recherche en Informatique et Télécommunication, Abidjan, Côte d'Ivoire
}

\begin{abstract}
Belief entropy, which represents the uncertainty measure between several pieces of evidence in the DempsterShafer framework, is attracting increasing interest in research. It has been used in many applications and is mainly based on the theory of evidence. To quantify uncertainty, several measures have been proposed in the literature. These measures, sometimes in extended or hybrid forms, use the Shannon entropy principle to determine uncertainty degree. However, the failure to consider the scale of the frame of discernment framework remains an open issue in quantifying uncertainty. In this paper, we propose a new uncertainty measure that takes into account the power set of the frame of discernment. After analysing the different existing methods, we show the performance and effectiveness of our proposed approach.
\end{abstract}

Keywords-Dempster Shafer Theory; Belief entropy; Uncertainty; Information management; Deng entropy

\section{INTRODUCTION}

In recent years, there have been increasing improvements in the management of uncertainty issues in information systems [1], [2], [3], [4]. Several theories including Shannon's entropy [5], probability theory [6], [7], possibility theory [8], [9] and Dempster-Shafer evidence theory [10], [11] have been developed. Dempster-Shafer theory in particular, provides effective tools for modelling and processing uncertain information [12], [13], [14], [15]. It has been widely used in various applications including information fusion [16], [17], decision making [18], [19], diagnosis and fault detection [20], [21], target recognition [22], [23], and so on. In applying this theory, many challenges are increasingly being addressed. We can mention, conflict management through different sources of information [24], [25], Consideration of the relationship between various pieces of evidence before the fusion step [26], [27], the problem in body of evidence $(B O E)$ generation [28], [29] and finally, the consideration of the frame of discernment (FOD) in uncertainty management [24], [30].

In the Dempster-Shafer framework, there are several hybrid or extended uncertainty measures. These measures use the Shannon entropy principle for the uncertainty quantifiying. Also, these measures include Hohle's confusion measure [31], Dubois \& Prade's weighted Hartley entropy [32], Yager's dissonance measure [33], Klir \& Ramer's discord measure [34], Klir \& Parviz's strife measure [35], George \& Pal's conflict measure [36], Deng entropy [37] and modified Deng entropy proposed by Zhou et al. [30].

In this work, we are mainly interested in Deng entropy and modified Deng entropy. The Deng entropy, a very effective measure, compared to various measures in some cases, has been used in several fields of application[38], [37]. However, one of the main limitations of this uncertainty measure is related to not considering the scale of FOD. To address this limit, some authors, including Zhou et al., have proposed a modified Deng measure [30]. Nevertheless, although effective in some cases, the problem related to the scale of $F O D$ is still perceptible. In this paper, we propose a new uncertainty measure by extending the modified Deng entropy. This new measure improves the performance of the measures proposed by Deng and Zhou takes into consideration the power set of the $F O D$. After analysing the different existing methods, we show the performance and effectiveness of our proposed approach.

The rest of this document is organized as follows : section 2 provides a brief overview of Dempster-Shafer theory and Shannon entropy. Section 3 presents some uncertainty measures in the Dempster-Shafer framework and and some limitations. Section 4 describe the new uncertainty measure. Section 5 presents, using numerical examples, the effectiveness of the new measure. The conclusion and some perspectives related to this work are presented in Section 6.

\section{PRELIMINARIES}

\section{A. Dempster-Shafer Theory}

Dempster-Shafer Theory [10], [11], also known as belief theory or evidence theory, has many advantages for processing uncertain information. We present some basic concepts related to this theory.

1) Formalism: Let $\Omega=\left\{\omega_{1}, \ldots, \omega_{N}\right\}$ a set of $N$ mutually exclusive and exhaustive events. $\Omega$ represents the frame of discernement $F O D$. A mass function is defined on the power set of $\Omega$, noted $2^{\Omega}$ with :

$$
2^{\Omega}=\left\{\emptyset,\left\{\omega_{1}\right\}, \ldots,\left\{\omega_{N}\right\}, \ldots,\left\{\omega_{1}, \ldots, \omega_{i}\right\}, \ldots, \Omega\right\}
$$

In $\Omega$, a mass function assigns to each subset a value between 0 and 1 representing its elementary belief mass defined by :

$$
m: 2^{\Omega} \rightarrow[0,1]
$$

Such as :

$$
m(\emptyset)=0 \quad \text { et } \quad \sum_{A \subseteq \Omega} m^{\Omega}(A)=1
$$

When $m(A)>0, A$ is called a focal element. Belief is the amount of trust that supports a hypothesis $A$ of the 
power set $2^{\Omega}$. It is most often called the Body Of Evidence $B O E$ or Basic Probability Assignment $B P A$ or Basic Belief Assignment $B B A[39]$ and is characterized by all the focal elements and their associated mass value (Eq.4):

$$
(\aleph, m)=\left\{(A, m(A)): A \in 2^{\Omega}, m(A)>0\right\}
$$

where $\aleph$ represents a subset of the power set $2^{\Omega}$ and each proposition $A \in \aleph$ are focal elements.

A $B O E$ can also be represented by its associated belief $\mathrm{Bel}$ and plausibility $P l$ functions defined as follows :

$$
\operatorname{Bel}(A)=\sum_{0 \neq B \subseteq A} m(B) \quad \text { and } \quad P l(A)=\sum_{B \cap A=\phi} m(B)
$$

2) Combination rules: In Dempster-Shafer theory, two independent mass functions, noted $m_{1}$ and $m_{2}$, can be combined with the Dempster's combination rule [40], defined as follows:

$$
m(A)=\frac{1}{1-k} \sum_{B \cap C=A} m_{1}(B) m_{2}(C)
$$

where $k$ represents the degree of conflict between $m_{1}$ and $m_{2} . k$ is defined as follows:

$$
k=\sum_{B \cap C=\phi} m_{1}(B) m_{2}(C)
$$

\section{B. Shannon entropy}

In information theory, Shannon entropy $\left(E_{s}\right)[5]$ is used to measure the volume of information in a system, process or message. This measure determines the expected value of the information contained in a message. The measure is defined as follows :

$$
E_{s}(m)=-\sum_{i=0}^{n} p_{i} \log _{b} p_{i}
$$

where $n$ represents the quantity or number of basic states, $p_{i}$ represents the probability of the state $i$ with $\sum_{i=0}^{n} p_{i}=1, b$ is the basis of the logarithm, it most often takes the value 2 .

\section{RELATED WORKS}

In this section, we present some uncertainty measures in the Dempster-Shafer framework. In these measures, $X$ represents $F O D, A$ and $B$ are the focal elements. $|A|$ refers to the cardinality of $A$.

\section{A. Uncertainty measures}

Some uncertainty measures are represented in the table I. These measures include Hohle's confusion measure [31], Dubois \& Prade's weighted Hartley entropy [32], Yager's dissonance measure [33], Klir \& Ramer's discord measure[34], Klir \& Parviz's strife measure [35], George \& Pal's conflict measure [36], Deng entropy [37] and modified Deng entropy proposed by Zhou et al. [30]. In this study, we are particularly interested in the entropies proposed by Deng and Zhou et al.

\section{B. Problem formulation}

In Demspter-Shafer theory, uncertain information should not only be modelled by mass functions, $F O D$ is also a source of uncertainty [38]. This paper recall this problem by using the Zhou et al.'s [30] example. Some measures such as Deng entropy $\left(E_{d}\right)$ and Modified Deng entropy $\left(E_{z}\right)$ are calculated.

Example 3.2: Consider two BOEs $m_{1}$ and $m_{2}$, representing respectively results of two reliable sensors in a target identification problem as follows :

$$
\begin{aligned}
& m_{1}: m_{1}(\{a, b\})=0.4, m_{1}(\{c, d\})=0.6 \\
& m_{2}: m_{2}(\{a, c\})=0.4, m_{2}(\{a, b\})=0.6
\end{aligned}
$$

Deng entropy is calculated as follows :

$$
\begin{aligned}
E_{d}\left(m_{1}\right) & =-\sum_{A \subseteq X} m_{1}(A) \log _{2}\left(\frac{m_{1}(A)}{2^{|A|}-1}\right) \\
& =-0.4 \times \log _{2}\left(\frac{0.4}{2^{2}-1}\right)-0.6 \times \log _{2}\left(\frac{0.6}{2^{2}-1}\right) \\
E_{d}\left(m_{1}\right) & =2.5559 \\
E_{d}\left(m_{2}\right) & =-\sum_{A \subseteq X} m_{2}(A) \log _{2}\left(\frac{m_{2}(A)}{2^{|A|}-1}\right) \\
& =-0.4 \times \log _{2}\left(\frac{0.4}{2^{2}-1}\right)-0.6 \times \log _{2}\left(\frac{0.6}{2^{2}-1}\right) \\
E_{d}\left(m_{2}\right) & =2.5559
\end{aligned}
$$

Despite the difference in FODs (i.e. $X_{1}=\{a, b, c, d\}$ et $\left.X_{2}=\{a, b, c\}\right)$, The results obtained from the Deng measure about the $B O E m_{1}$ are similar to the uncertainty measure of $\mathrm{BOE} \mathrm{m}_{2}$. Intuitively, the uncertainty measure of $\mathrm{BOE}$ $m_{1}$ should be bigger than that of $B O E m_{2}$. This, because the $F O D$ related to the $B O E m_{1}$ contains more elements than the $F O D$ related to the $B O E m_{2}$. At this main limitation, some authors, notably Zhou et al. [30] have proposed the modified Deng entropy.

Modified Deng entropy is calculated as follows :

$$
\begin{aligned}
E_{z}\left(m_{1}\right) & =-\sum_{A \subseteq X} m_{1}(A) \log _{2}\left(\frac{m_{1}(A)}{2^{|A|}-1} e^{\frac{|A|-1}{|X|}}\right) \\
& =-0.4 \times \log _{2}\left(\frac{0.4}{2^{2}-1} e^{\frac{2-1}{4}}\right)-0.6 \times \log _{2}\left(\frac{0.6}{2^{2}-1} e^{\frac{2-1}{3}}\right) \\
E_{z}\left(m_{1}\right) & =2.1952 \\
E_{z}\left(m_{2}\right) & =-\sum_{A \subseteq X} m_{2}(A) \log _{2}\left(\frac{m_{2}(A)}{2^{|A|}-1} e^{\frac{|A|-1}{|X|}}\right) \\
E_{z}\left(m_{2}\right) & =-0.4 \times \log _{2}\left(\frac{0.4}{2^{2}-1} e^{\frac{2-1}{3}}\right)-0.6 \times \log _{2}\left(\frac{0.6}{2^{2}-1} e^{\frac{2-1}{3}}\right)
\end{aligned}
$$

As can be seen, the modified Deng entropy gives almost different results for each $B O E$. In this measure, we can see that the degree of uncertainty calculated from the different $B O E s$ is reduced compared to the Deng measure. However, although the modified Deng entropy takes into account the 
TABLE I. SOME Uncertainty MEAsures

\begin{tabular}{l|c}
\hline Measures & Expression \\
\hline \hline Hohle's confusion measure [31] & $C_{h}(m)=-\sum_{A \subseteq X} m(A) \log _{2} \operatorname{Bel}(A)$ \\
\hline Yager's dissonance measure [33] & $E_{y}(m)=-\sum_{A \subseteq X} m(A) \log _{2} P l(A)$ \\
\hline Dubois \& Prade's weighted Hartley entropy [32] & $E_{d p}(m)=-\sum_{A \subseteq X} m(A) \log _{2}|A|$ \\
\hline Klir \& Ramer's discord measure [34] & $D_{k r}(m)=-\sum_{A \subseteq X} m(A) \log _{2} \sum_{B \subseteq X} m(B) \frac{|A \cap B|}{|B|}$ \\
\hline Klir \& Parviz's strife measure [35] & $S_{k p}(m)=-\sum_{A \subseteq X} m(A) \log _{2} \sum_{B \subseteq X} m(B) \frac{|A \cap B|}{|A|}$ \\
\hline George \& Pal's conflict measure [36] & $C_{g p}(m)=-\sum_{A \subseteq X} m(A) \log _{2} \sum_{B \subseteq X} m(B)\left[1-\frac{|A \cap B|}{|A \cup B|}\right]$ \\
\hline Deng entropy [37] & $E_{d}(m)=-\sum_{A \subseteq X} m(A) \log _{2}\left(\frac{m(A)}{2^{|A|}-1}\right)$ \\
\hline Modified Deng entropy [30] & $E_{z}(m)=-\sum_{A \subseteq X} m(A) \log _{2}\left(\frac{m(A)}{2^{|A|}-1} e^{\frac{|A|-1}{|X|}}\right)$ \\
\hline
\end{tabular}

number of elements in the $F O D$, this measure often gives counter-intuitive measures. We present another problem in example 3.3.

Example 3.3 : Consider the BOEs $m_{3}$ and $m_{4}$ in the FOD $X=\{a, b\}$ as follows :

$$
\begin{gathered}
m_{3}: m_{3}(\{a, b\})=1 \\
m_{4}: m_{4}(\{a\})=m_{4}(\{b\})=0.5
\end{gathered}
$$

Modified Deng entropy is calculated as follows :

$$
\begin{gathered}
E_{z}\left(m_{3}\right)=-1 \times \log _{2}\left(\frac{1}{2^{2}-1} e^{\frac{2-1}{2}}\right)=0.8636 \\
E_{z}\left(m_{4}\right)=-0.5 \times \log _{2}\left(\frac{0.5}{2^{1}-1} e^{\frac{1-1}{2}}\right)-0.5 \times \log _{2}\left(\frac{0.5}{2^{1}-1} e^{\frac{1-1}{2}}\right)=1
\end{gathered}
$$

Deng entropy is calculated as follows :

$$
\begin{gathered}
E_{d}\left(m_{3}\right)=-1 \times \log _{2}\left(\frac{1}{2^{2}-1}\right)=1.5850 \\
E_{d}\left(m_{4}\right)=-0.5 \times \log _{2}\left(\frac{0.5}{2^{1}-1}\right)-0.5 \times \log _{2}\left(\frac{0.5}{2^{1}-1}\right)=1
\end{gathered}
$$

As can be seen, example 3.3 defines two BOEs $m_{3}$ and $m_{4}$ where $B O E m_{3}$ represents a case of total uncertainty. Intuitively, the uncertainty level of the $B O E m_{3}$ must be bigger than that of the $B O E m_{4}$, which is in contradiction with the modified Deng measure $\left(E_{z}\right)$. However, the Deng measure $\left(E_{d}\right)$ better distinguishes total uncertainty with an uncertainty measure of $B O E m_{3}$ bigger than that of $B O E m_{4}$.

Thus, after analyzing examples 3.2 and 3.3 , it can be seen that example 3.2 presents a case of variable $F O D$ with the same number of elements in the focal elements. And example 3.3 presents a case where the $F O D$ does not vary. In contrast, in example 3.3, the measure proposed by Deng has better results compared to the Zhou et al. results. Therefore, how to quantify optimally uncertainty by taking into account the limits observed in the measures proposed by Deng and Zhou et al.?

To solve this problem, we propose a new uncertainty measure by extending the measures proposed by Deng and Zhou et al.

\section{New Uncertainty Measure}

In the Dempster-Shafer framework, the new uncertainty measure $\left(E_{N m}\right)$ we propose is as follows:

$$
E_{N m}(m)=-\sum_{A \subseteq X} m(A) \log _{2}\left(\frac{m(A)}{2^{|A|}-1} e^{\frac{|A|-1}{2|X|}}\right)
$$

where $m$ is the mass function defined on $X . A$ is the focal element of $X$ and $|A|$ represents the cardinality of $A$.The particularity of this measure is that it takes into account the number of elements of the power set represented by $2^{|X|}$.

A simple transformation of the new entropy is as follows:

$$
\begin{aligned}
& E_{N m}(m)=\sum_{A \subseteq X} m(A) \log _{2}\left(2^{|A|}-1\right)-\sum_{A \subseteq X} m(A) \log _{2} m(A) \\
& -\sum_{A \subseteq X} m(A) \log _{2} e^{\frac{|A|-1}{2^{|X|}}}
\end{aligned}
$$

As can be seen in this expression, the first two terms refer to Deng entropy [37]. These are respectively the measure of the total non-specificity in the mass function $m$, and the measure of the discord of the mass function between focal elements. The third term, the exponential factor, $e^{\frac{\mid A-1}{2|X|}}$, is the main factor in this contribution. The choice of this factor is based on the exponential factor (i.e. $e^{\frac{\mid A-1}{|X|}}$ ) proposed by Zhou et al. [30], which represents the measure of uncertain information in a $B O E$. Compared to the Zhou et al. measure, the new measure takes into account the number of elements in the power set represented by $2^{|X|}$. Thus, the new measure is intended to be more generic in resolving the limitations of the measures proposed by Deng and Zhou et al. in examples 3.2 and 3.3 respectively. Let's go back to example 3.2, the new 
entropy is as follows:

$$
\begin{aligned}
E_{N m}\left(m_{1}\right) & =-\sum_{A \subseteq X} m_{1}(A) \log _{2}\left(\frac{m_{1}(A)}{2^{|A|}-1} e^{\frac{|A|-1}{2^{|X|}}}\right) \\
& =-0.4 \times \log _{2}\left(\frac{0.4}{2^{2}-1} e^{\frac{2-1}{2^{4}}}\right)-0.6 \times \log _{2}\left(\frac{0.6}{2^{2}-1} e^{\frac{2-1}{2^{3}}}\right) \\
E_{N m}\left(m_{1}\right) & =2.4657 \\
E_{N m}\left(m_{2}\right) & =-\sum_{A \subseteq X} m_{2}(A) \log _{2}\left(\frac{m_{2}(A)}{2^{|A|}-1} e^{\frac{|A|-1}{2^{|X|}}}\right) \\
& =-0.4 \times \log _{2}\left(\frac{0.4}{2^{2}-1} e^{\frac{2-1}{2^{3}}}\right)-0.6 \times \log _{2}\left(\frac{0.6}{2^{2}-1} e^{\frac{2-1}{2^{3}}}\right) \\
E_{N m}\left(m_{2}\right) & =2.3755
\end{aligned}
$$

The results of the different uncertainty measures of the two (BOEs) $m_{1}$ and $m_{2}$ are summarized in the table II.

TABle II. Uncertainty Measures Calculated in EXample 3.2

\begin{tabular}{|c|c|c|c|}
\hline BOEs & Deng entropy & $\begin{array}{c}\text { Modified } \\
\text { Deng entropy }\end{array}$ & New entropy \\
\hline$m_{1}$ & 2.5559 & 2.1952 & 2.4657 \\
\hline$m_{2}$ & 2.5559 & 2.0750 & 2.3755 \\
\hline
\end{tabular}

In this table, like Zhou et al.'s proposed measure, the new measure gives different measures for each of BOEs $m_{1}$ and $m_{2}$. However, the new measure gives bigger values compared to those of Zhou et al. Table III takes the measures from table II, provides the distance $d\left(m_{1}, m_{2}\right)$ between the measures of $B O E s m_{1}$ and $m_{2}$ to determine the observed information loss between these BOEs. Finally, the BOE $m 1$ measure is added to the calculated distance. In this case, the new measure compared to the Zhou et al. measure, takes into account the loss of perceived information in the Deng measure.

TABLE III. EfFectiveness of the New Measure

\begin{tabular}{|c|c|c|c|}
\hline BOEs & $\begin{array}{c}\text { Deng entropy } \\
{[37]}\end{array}$ & $\begin{array}{c}\text { Modified } \\
\text { Deng entropy } \\
{[30]}\end{array}$ & $\begin{array}{c}\text { New } \\
\text { entropy }\end{array}$ \\
\hline$m_{1}$ & 2.5559 & 2.1952 & 2.4657 \\
\hline$m_{2}$ & 2.5559 & 2.0750 & 2.3755 \\
\hline$d\left(m_{1}, m_{2}\right)$ & 0 & 0.1202 & 0.0902 \\
\hline $\begin{array}{c}m_{1}+ \\
d\left(m_{1}, m_{2}\right)\end{array}$ & 2.5559 & 2.3154 & 2.5559 \\
\hline
\end{tabular}

Thus, using the example 3.3, the new measure of the different BOEs $m_{3}$ and $m_{4}$ is as follows :

$$
\begin{aligned}
& E_{N m}\left(m_{3}\right)=-1 \times \log _{2}\left(\frac{1}{2^{2}-1} e^{\frac{2-1}{2^{2}}}\right)=1.2243 \\
& E_{N m}\left(m_{4}\right)=-0.5 \times \log _{2}\left(\frac{0.5}{2^{1}-1} e^{\frac{1-1}{2^{2}}}\right)-0.5 \times \log _{2}\left(\frac{0.5}{2^{1}-1} e^{\frac{1-1}{2^{2}}}\right) \\
& E_{N m}\left(m_{4}\right)=1
\end{aligned}
$$

The results of the (BOEs) $m_{3}$ and $m_{4}$ are summarized in the table IV. In this table, the new entropy $\left(E_{N m}\right)$ is also represented. And as can be seen, only the entropy proposed by Zhou et al. gives counter-intuitive results. In this case of example where the $F O D$ does not vary, the new measure is close to the merits of the Deng measure.

TABLE IV.

Uncertainty Measures Calculated in Example 3.3

\begin{tabular}{|c|c|c|c|}
\hline BOEs & $\begin{array}{c}\text { Deng entropy } \\
\text { [37] }\end{array}$ & $\begin{array}{c}\text { Modified } \\
\text { Deng } \\
\text { Entropy [30] }\end{array}$ & New Entropy \\
\hline$m_{3}$ & 1.5850 & 0.8636 & 1.2243 \\
\hline$m_{4}$ & 1 & 1 & 1 \\
\hline
\end{tabular}

Thus, the proposed new measure responds to the limitations of Deng and Zhou et al. by taking a more generic character and an efficient quantification of uncertainty.

\section{Proof And Discussions}

In this section, we first present some fundamental properties of the new uncertainty measure. Then, using numerical examples, we show the concordance of the new entropy with some basic entropy including Shannon's entropy $\left(E_{s}\right)$, Deng entropy $\left(E_{d}\right)$ and the entropy proposed by Zhou et al. $\left(E_{z}\right)$. Finally, we discuss the superiority of the new entropy compared to the above-mentioned entropies.

\section{A. Concordance with Shanon entropy}

The proposed entropy measure is identical to the basic entropy, the Shannon entropy (Eq. 17), when we have a Bayesian mass function (i.e. a single element in the $B O E$ or $|A| \equiv 1)$ as follows.

$$
\begin{aligned}
E_{N m}(m) & =-\sum_{A \subseteq X} m(A) \log _{2}\left(\frac{m(A)}{2^{|A|}-1} e^{\frac{|A|-1}{2^{|X|}}}\right) \\
& =-\sum_{A \subseteq X} m(A) \log _{2}\left(\frac{m(A)}{2^{1}-1} e^{\frac{1-1}{2^{|X|}}}\right) \\
& =-\sum_{A \subseteq X} m(A) \log _{2} m(A) \\
E_{N m}(m) & =E_{s}(m)
\end{aligned}
$$

In addition, another proof of concordance of the new entropy with different uncertainty measures is provided in the case where the $(F O D)$ has only one element (1.e. total uncertainty case) as shown in the following example.

Example 5.1 : Consider an information processing system in which information $I$ reported by a sensor has a belief equal to one hundred percent. In $X=\{I\}$, the mass function can be noted as $m(\{I\})=1$. The calculation of the entropies of Shannon $\left(E_{s}\right)$, Deng $\left(E_{d}\right)$, Zhou et al $\left(E_{z}\right)$ and the new entropy measure $\left(E_{N m}\right)$ are defined as follows:

$$
\begin{gathered}
E_{s}(m)=-1 \times \log _{2} 1=0 \\
E_{d}(m)=-1 \times \log _{2} \frac{1}{2^{1}-1}=0 \\
E_{z}(m)=-1 \times \log _{2}\left(\frac{1}{2^{1}-1} e^{\frac{1-1}{1}}\right)=0 \\
E_{N m}(m)=-1 \times \log _{2}\left(\frac{1}{2^{1}-1} 2^{\frac{1-1}{2^{1}}}\right)=0
\end{gathered}
$$




\section{B. Superiority of the new uncertainty measure}

To show the superiority of the new uncertainty measure, recall the example mentioned in [37].

Exemple 5.3 : Consider a mass function $m$ in a $F O D X$ such that : $X=\{1,2,3,4,5,6,7,8,9,10,11,12,13,14,15\}$, $m(\{6\})=0.05, m(\{3,4,5\})=0.05, m(E)=0.8$ et $m(X)=$ 0.1 . $E$ represents a subset of elements varying from 1 to 14 as shown in the table $\mathrm{V}$.

TABle V. Entropy Measures with a Subset $E$ of Variable ELEMENTS

\begin{tabular}{|c|c|c|c|}
\hline Elements in $E$ & $\begin{array}{c}\text { Deng } \\
\text { Entropy } \\
{[37]}\end{array}$ & $\begin{array}{c}\text { Modified } \\
\text { Deng } \\
\text { entropy } \\
{[30]}\end{array}$ & $\begin{array}{c}\text { New } \\
\text { Entropy }\end{array}$ \\
\hline$E=\{1\}$ & 2.6623 & 2.5180 & 2.6622 \\
\hline$E=\{1,2\}$ & 3.9303 & 3.7090 & 3.9302 \\
\hline$E=\{1,2,3\}$ & 4.9082 & 4.6100 & 4.9080 \\
\hline$E=\{1, \ldots, 4\}$ & 5.7878 & 5.4127 & 5.7876 \\
\hline$E=\{1, \ldots, 5\}$ & 6.6256 & 6.1736 & 6.6254 \\
\hline$E=\{1, \ldots, 6\}$ & 7.4441 & 6.9151 & 7.4439 \\
\hline$E=\{1, \ldots, 7\}$ & 8.2532 & 7.6473 & 8.2530 \\
\hline$E=\{1, \ldots, 8\}$ & 9.0578 & 8.3749 & 9.0575 \\
\hline$E=\{1, \ldots, 9\}$ & 9.8600 & 9.1002 & 9.8597 \\
\hline$E=\{1, \ldots, 10\}$ & 10.6612 & 9.8244 & 10.6608 \\
\hline$E=\{1, \ldots, 11\}$ & 11.4617 & 10.5480 & 11.4613 \\
\hline$E=\{1, \ldots, 12\}$ & 12.2620 & 11.2714 & 12.2616 \\
\hline$E=\{1, \ldots, 13\}$ & 13.0622 & 11.9946 & 13.0617 \\
\hline$E=\{1, \ldots, 14\}$ & 13.8622 & 12.7177 & 13.8617 \\
\hline
\end{tabular}

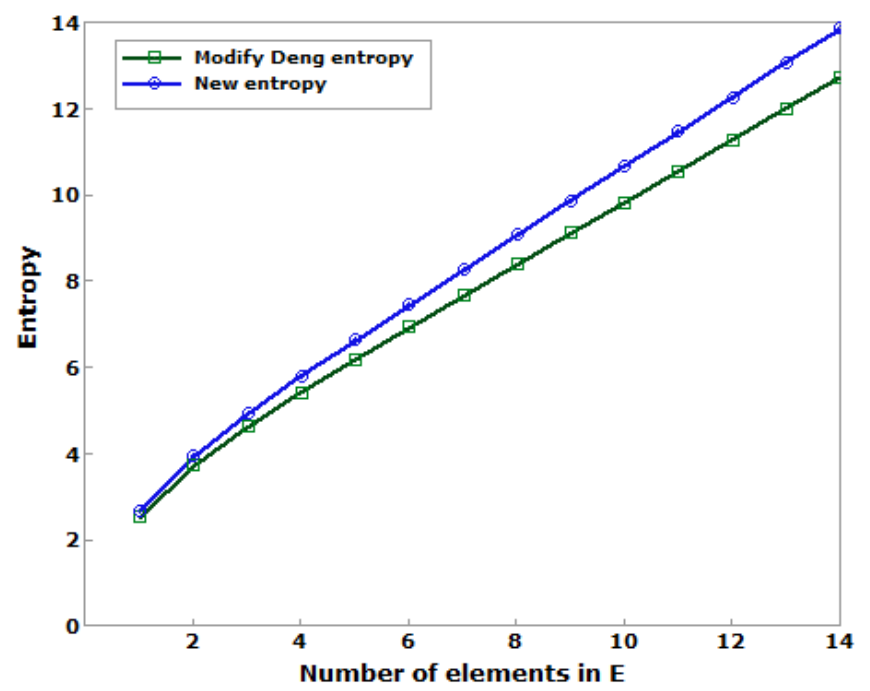

Fig. 1. Comparison between the entropy proposed by Zhou et al. and the new entropy
As can be seen in Figure 1, the new and modified entropy of Deng proposed by Zhou et al. increase monotonously with increasing size of the subset $E$. However, the values of the new entropy are significantly bigger than that of Zhou et al. measure. As shown in example 3.3. the measure proposed by Zhou et al. records losses of information especially in such a case where FOD that does not vary. Moreover, the new entropy gives results almost identical to the Deng measurement (figure 2), hence the effectiveness of the approach when the $F O D$ does not change.

Moreover, the new entropy gives results almost identical to the Deng measure (figure 2), hence the effectiveness of the new approach when the FOD does not change. The new measure does not differ from the merits of the Deng measure.

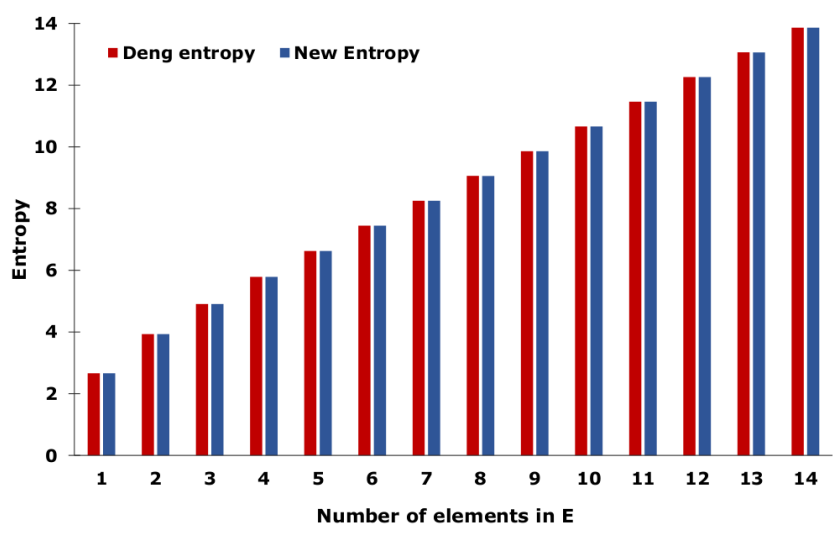

Fig. 2. Comparison between Deng entropy and new entropy

Figure 3 shows the degree of uncertainty of the $B O E$ using other different uncertainty measures including Hohle's confusion measure [31], Dubois \& Prade's weighted Hartley entropy [32], Yager's dissonance measure [33], Klir \& Ramer's discord measure [34], Klir \& Parviz's strife measure [35], George \& Pal's conflict measure [36]. In this figure, we can observe that only the entropies of Dubois \& Prade [32], Deng [37], Zhou [30], and the new entropy increase monotonously with the increase in the size of subset $E$. Also with the increase in size of $E$, there is either a declination or a change in the pace of other uncertainty measures. Hence the effectiveness of the new measure.

\section{CONCLUSION}

Quantifying uncertainty in information systems is very important for evaluating the quality of information. Several methods based on entropy of beliefs have been proposed in the literature, but these give counter-intuitive results, particularly in the case of variable $F O D$ with $B O E$. In this paper, we have proposed a new measure to address these deficiencies. This measure extends the measures proposed by Deng and Zhou et al. From numerical examples and mathematical properties, we have shown the effectiveness of the new measure which gives more information in the power set of the FOD. Our future studies will focus on the actual application of the news in several areas including decision making, fault diagnosis and detection, and so on. 


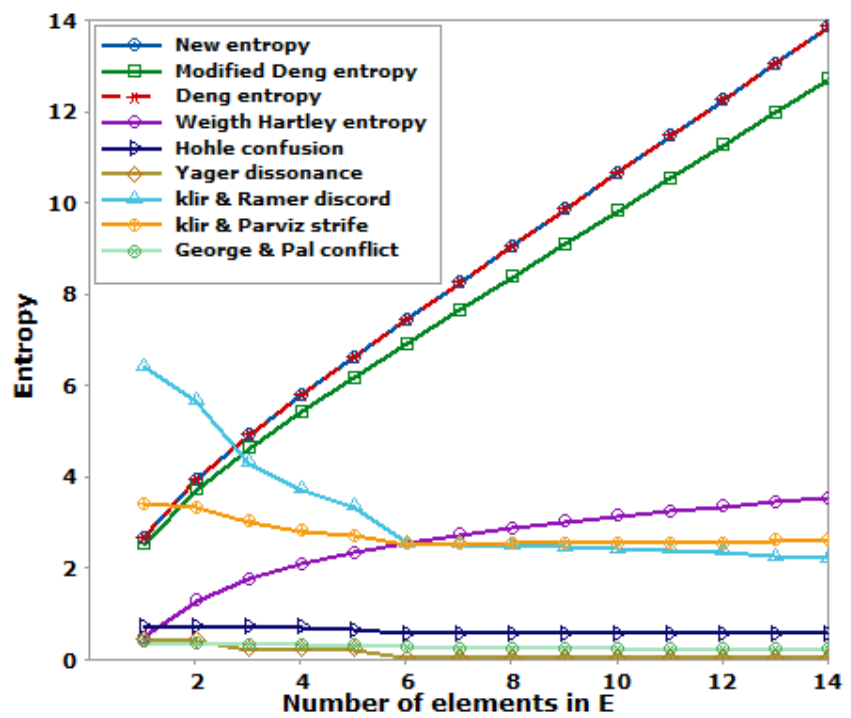

Fig. 3. Comparison with other uncertainty mesures

\section{REFERENCES}

[1] Y. Deng, "D numbers: theory and applications," Journal of Information \&Computational Science, vol. 9, no. 9, pp. 2421-2428, 2012.

[2] G. J. Klir, "Generalized information theory," Fuzzy Sets and Systems, vol. 40, no. 1, pp. 127-142, Mar. 1991.

[3] L. A. Zadeh, "Toward a generalized theory of uncertainty (GTU)an outline," Information Sciences, vol. 172, no. 1, pp. 1-40, 2005.

[4] I. A. T. Hashem, I. Yaqoob, N. B. Anuar, S. Mokhtar, A. Gani, and S. Ullah Khan, "The rise of big data on cloud computing: Review and open research issues," Information Systems, vol. 47, pp. 98-115, Jan. 2015.

[5] C. E. Shannon, "A note on the concept of entropy," Bell System Tech. $J$, vol. 27, no. 3, pp. 379-423, 1948.

[6] W. Feller, An introduction to probability theory and its applications. John Wiley \& Sons, 2008, vol. 2.

[7] J. M. Steele, "Probability theory: Formal," 2015.

[8] D. Dubois and H. Prade, "Possibility Theory," in Computational Complexity: Theory, Techniques, and Applications, R. A. Meyers, Ed. New York, NY: Springer New York, 2012, pp. 2240-2252.

[9] L. N. Kanal and J. F. Lemmer, Uncertainty in artificial intelligence, 2014, vol. 4.

[10] A. P. Dempster, "Upper and Lower Probabilities Induced by a Multivalued Mapping," The Annals of Mathematical Statistics, vol. 38, no. 2, pp. 325-339, 1967.

[11] G. Shafer, A Mathematical Theory of Evidence. Princeton University Press, 1976.

[12] D. Harmanec and G. J. Klir, "Measuring Total Uncertainty in DempsterShafer Theory: A Novel Approach," International Journal of General Systems, vol. 22, no. 4, pp. 405-419, 1994.

[13] R. D. Shachter, L. Kanal, M. Henrion, and J. F. Lemmer, Uncertainty in Artificial Intelligence 5, 2017, vol. 10.

[14] H. B. Sta, "Quality and the efficiency of data in Smart-Cities," Future Generation Computer Systems, vol. 74, pp. 409-416, 2017.

[15] A. Gaur, B. Scotney, G. Parr, and S. McClean, "Smart city architecture and its applications based on iot," Procedia computer science, vol. 52, pp. 1089-1094, 2015.

[16] H. Wu, M. Siegel, R. Stiefelhagen, and J. Yang, "Sensor fusion using Dempster-Shafer theory [for context-aware HCI]," in Instrumentation and Measurement Technology Conference, 2002. IMTC/2002. Proceedings of the 19th IEEE, vol. 1. IEEE, 2002, pp. 7-12.

[17] B. Khaleghi, A. Khamis, F. O. Karray, and S. N. Razavi, "Multisensor data fusion: A review of the state-of-the-art," Information fusion, vol. 14, no. 1, pp. 28-44, 2013.
[18] M. Bauer, "Approximation algorithms and decision making in the Dempster-Shafer theory of evidenceAn empirical study," International Journal of Approximate Reasoning, vol. 17, no. 2-3, pp. 217-237, 1997.

[19] D. Yong, S. WenKang, Z. ZhenFu, and L. Qi, "Combining belief functions based on distance of evidence," Decision support systems, vol. 38, no. 3, pp. 489-493, 2004.

[20] O. Basir and X. Yuan, "Engine fault diagnosis based on multi-sensor information fusion using DempsterShafer evidence theory," Information Fusion, vol. 8, no. 4, pp. 379-386, 2007.

[21] A. Paksoy and M. Göktürk, "Information fusion with dempster-shafer evidence theory for software defect prediction," Procedia Computer Science, vol. 3, pp. 600-605, 2011.

[22] D. M. Buede and P. Girardi, "A target identification comparison of Bayesian and Dempster-Shafer multisensor fusion," IEEE Transactions on Systems, Man, and Cybernetics-Part A: Systems and Humans, vol. 27, no. 5, pp. 569-577, 1997.

[23] M. Beynon, B. Curry, and P. Morgan, "The dempster-shafer theory of evidence: an alternative approach to multicriteria decision modelling," Omega, vol. 28, no. 1, pp. 37-50, 2000.

[24] E.-P. Lim, J. Srivastava, and S. Shekhar, "An evidential reasoning approach to attribute value conflict resolution in database integration," IEEE Transactions on Knowledge and Data Engineering, vol. 8, no. 5, pp. 707-723, 1996.

[25] J. Yang, H.-Z. Huang, Q. Miao, and R. Sun, "A novel information fusion method based on Dempster-Shafer evidence theory for conflict resolution," Intelligent Data Analysis, vol. 15, no. 3, pp. 399-411, 2011.

[26] K. Yamada, "A new combination of evidence based on compromise," Fuzzy sets and Systems, vol. 159, no. 13, pp. 1689-1708, 2008.

[27] L. A. Zadeh, "A simple view of the Dempster-Shafer theory of evidence and its implication for the rule of combination," AI magazine, vol. 7, no. 2, p. 85, 1986

[28] P. Xu, X. Su, S. Mahadevan, C. Li, and Y. Deng, "A non-parametric method to determine basic probability assignment for classification problems," Applied intelligence, vol. 41, no. 3, pp. 681-693, 2014.

[29] W. Jiang, J. Zhan, D. Zhou, and X. Li, "A method to determine generalized basic probability assignment in the open world," Mathematical Problems in Engineering, vol. 2016, 2016.

[30] D. Zhou, Y. Tang, and W. Jiang, "A modified belief entropy in Dempster-Shafer framework," PLOS ONE, vol. 12, no. 5, p. e0176832, 2017.

[31] U. HOHLE, "Entropy with respect to Plausibility Measures," Proc. of 12th IEEE Int. Symp. on Multiple Valued Logic, Paris, 1982, 1982.

[32] D. Dubois and H. Prade, "Properties of measures of information in evidence and possibility theories," Fuzzy Sets and Systems, vol. 24, no. 2, pp. 161-182, 1987.

[33] R. R. Yager, "Entropy and Specificity in a Mathematical Theory of Evidence," International Journal of General Systems, vol. 9, no. 4, pp. 249-260, 1983.

[34] G. J. Klir and A. Ramer, "Uncertainty in the Dempster-Shafer Theory: A Critical Re-examination," International Journal of General Systems, vol. 18, no. 2, pp. 155-166, 1990.

[35] G. J. Klir and B. Parviz, "A note on the Measure of Discord," in Uncertainty in Artificial Intelligence, D. Dubois, M. P. Wellman, B. D'Ambrosio, and P. Smets, Eds. Morgan Kaufmann, Jan. 1992, pp. 138-141.

[36] T. George and N. R. Pal, "Quantification of Conflict in DempsterShafer Framework: A new approach," International Journal of General Systems, vol. 24, no. 4, pp. 407-423, 1996.

[37] Y. Deng, "Deng entropy," Chaos, Solitons \& Fractals, vol. 91, pp. 549553, Oct. 2016.

[38] J. Wang, F. Xiao, X. Deng, L. Fei, and Y. Deng, "Weighted Evidence Combination Based on Distance of Evidence and Entropy Function," International Journal of Distributed Sensor Networks, vol. 12, no. 7, p. 3218784, Jul. 2016.

[39] P. P. Shenoy and G. Shafer, "Axioms for Probability and Belief-Function Propagation," in Classic Works of the Dempster-Shafer Theory of Belief Functions, ser. Studies in Fuzziness and Soft Computing, R. R. Yager and L. Liu, Eds. Berlin, Heidelberg: Springer Berlin Heidelberg, 2008, pp. $499-528$. 
[40] T. Denoeux, "Conjunctive and disjunctive combination of belief functions induced by nondistinct bodies of evidence," Artificial Intelligence, vol. 172, pp. 234-264, 2008. 